\title{
Pupillary Reaction in Children with Recurrent Abdominal Pain
}

\author{
JOHN APLEY, DIANA R. HASLAM, and C. GRANT TULLOH \\ From United Bristol Hospitals, and Department of Psychology, Bristol University
}

\begin{abstract}
Apley, J., Haslam, D. R., and Grant Tulloh, C. (1971). Archives of Disease in Childhood, 46, 337. Pupillary reaction in children with recurrent abdominal pain. An investigation was carried out with 3 groups of children to compare pupillary reactions (as an index of autonomic function) in response to stress induced by the cold pressor test. It was found that normal pupillary reactions are modified in children who have recurrent abdominal pain without physical cause and also in children with behaviour problems. It is suggested that disturbances of autonomic function merit further investigation in psychosomatic and emotional disorders, both as a method of diagnosis and to help elucidate the underlying mechanisms whereby symptoms may be produced.
\end{abstract}

Recurrent abdominal pain affects 1 child in 10 and so is one of the common disorders of childhood. Though an organic cause is occasionally found, there is a great deal of clinical evidence to indicate that this symptom is commonly a somatic expression of emotional disturbance (Apley, 1964). As in other psychogenic disorders, one pathway by which the disturbance is expressed in physical terms appears to be the autonomic nervous system.

One of the methods of studying autonomic function is by means of pupillometry, and this was used by Rubin, Barbero, and Sibinga (1967) to determine whether children with recurrent abdominal pain are characterized by autonomic dysfunction. These authors found that a group of children suffering from this disorder took significantly longer than a 'normal' control group to recover from stress induced by the cold pressor test; that is, they showed a slower decrement in the pupillary dilatation resulting from this stress than did the 'normal' children.

The observation that rate of recovery after stress differentiated the 2 groups has important implications. It was decided, therefore, to carry out an investigation similar to that of Rubin et al. (1967) but with some modifications. To this end, an experiment was designed to measure pupillary reactions in children under the following conditions: (1) constriction in response to light; (2) dilatation in the dark at rest; (3) dilatation in the dark under stress (expected to be greater than (2)); (4) constriction during recovery from stress.

Received 4 January 1970.

\section{Method}

Pupillometry is a method whereby the reactions of the pupil are measured under controlled conditions. The pupillary light reflex is subserved by parasympathetic and sympathetic branches of the autonomic nervous system. The iris contains 2 muscles, the sphincter pupillae and the dilator pupillae. The sphincter pupillae is supplied by parasympathetic fibres from the oculomotor nerve, and it is generally accepted that these fibres are responsible for pupillary constriction in response to light. The dilator pupillae receives its fibres from the superior cervical sympathetic ganglion.

Subjects. Fifty subjects made up 3 groups, 2 experimental and 1 control. Children with recurrent abdominal pain without physical disease formed 1 experimental group, called the RAP group. The other was a mixed group of children with behaviour problems, called the 'emotional' group. The control group consisted of children who were undergoing treatment for non-acute complaints such as nephritis.

Table I shows the number and the sex distribution of children in each group, and Table II shows the mean age and range in years for the 3 groups.

TABLE I

Number of Male and Female Children in Three Groups

\begin{tabular}{lc|c|c|c}
\hline & Control & RAP & Emotional & Total \\
\hline Male & 12 & 6 & 8 & 26 \\
Female & 8 & 10 & 6 & 24 \\
\hline Total & 20 & 16 & 14 & 50 \\
\hline
\end{tabular}


TABLE II

Mean Age and Range in Years of Children in Three Groups

\begin{tabular}{l|c|c|c}
\hline & Control & RAP & Emotional \\
\hline \begin{tabular}{l|r} 
Mean age $(y r)$ \\
Range
\end{tabular} & $\begin{aligned} 12 \cdot 05 \\
8-18\end{aligned}$ & $\begin{array}{r}9 \cdot 81 \\
5-13\end{array}$ & $\begin{array}{r}10 \cdot 93 \\
8-13\end{array}$ \\
\hline
\end{tabular}

Apparatus and procedure. The photographic apparatus consisted of 4 parts (see Fig. 1). (1) A fixation light run off a $1 \frac{1}{2}$ volt battery. (2) Infrared illumination produced by a 500 watt photoflood lamp and an infrared filter $\times 10$. An infrared sensitive film was used.

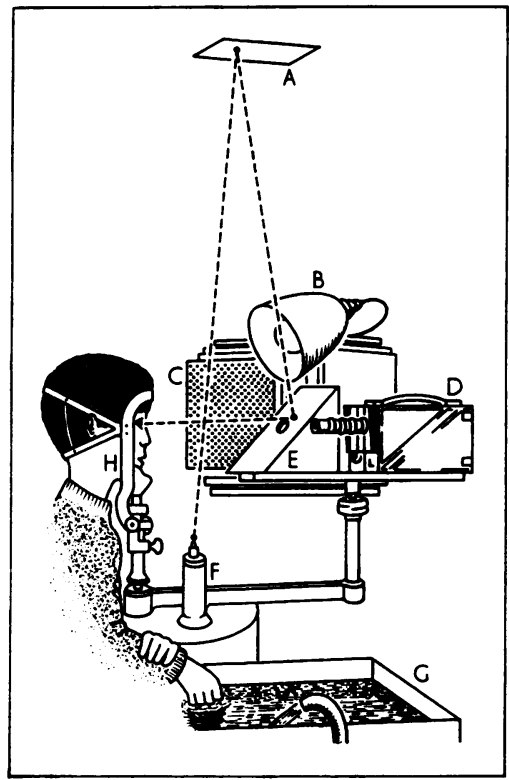

Fig.-Pupillometry apparatus. A-mirror (1); Badapting light; $C$-photoflood lamp with infrared filter and cooling fan; $D-16 \mathrm{~mm}$ cine camera with infrared sensitive film; $E$-mirror (2); $F$-fixation light; $G$-cold water bath; and $H$-head rest.

(3) An adapting light consisting of a 60 watt bulb emitting 50 foot-candles of illumination at the eye. (4) A $16 \mathrm{~mm}$ cine camera which was operated on single frames. Timing was by means of a stop-watch. The distance from the fixation light to the eye was $23 \mathrm{ft}$, obtained by a system of mirrors.

The differences between this apparatus and that used by Rubin were as follows. (1) The fixation light was much more distant in order to eliminate effects on the pupil due to accommodation and convergence. (2) Infrared illumination was used to eliminate the disturbing effects on the eye of repeated flash photographs.

Measurements were made as follows. Prints were $\vec{\nexists}$ made from $16 \mathrm{~mm}$ film and were enlarged to twice $\stackrel{0}{\rightarrow}$ life-size (a scale was photographed against the patient's 0 eye at the end of each series to check this). Each print 흘 was measured in 3 ways: (a) with a ruler; (b) with a $\overline{\bar{c}}$ pair of dividers which was then placed on a ruler; (c) with a Pifco magnascope. The measurements were made to the nearest $0.5 \mathrm{~mm}$ and then (to reduce them again to life-size) divided by 2 , and so were to the nearest $0.25 \mathrm{~mm}$.

An attempt was made to put the children at their ease on entering the pupillometry room, and they were given time to become familiar with the environment while they were getting comfortably seated. Each child sat at a table with his head strapped in the headrest, and all measurements were made in the darkened room.

(1) Rest condition. After 5 seconds in the dark, the adapting light was focused on the child's left eye for 10 seconds, and then a photograph was taken after 5 and again after 10 seconds. There followed a 5-minute dark-adaptation period. During this time an infrared photograph was taken every 5 seconds for the first minute, and then at the end of each successive minute.

(2) Stress condition. The adapting light was then focused for a further 10 seconds on the left eye and a photograph was taken after 5 seconds and again after 10 seconds. Immediately on extinction of the adapting light each child had his left hand placed in a cold water bath maintained at $5^{\circ} \pm 2{ }^{\circ} \mathrm{C}$ and it was kept immersed for 1 minute. During this time an infrared photograph was taken every 5 seconds. The hand was then removed from the cold water and dried very gently so as not to interfere with the measurements. After the cold water immersion there followed a 10-minute 'recovery' period. During the first minute of this time a photograph was taken every 5 seconds. After this, one was taken at the end of each successive minute for the next 9 minutes.

\section{Results and Discussion}

'Rest' condition and 'stress' condition. The mean values for pupil diameter for the 3 groups, after 1 minute in the dark (rest condition) and after 1 minute of the cold pressor test (stress condition), are shown in Table III. A Wilcoxon matchedpairs signed-ranks test showed that only for the control group was there a significant difference between rest and stress values $(P<0 \cdot 01$, two-tailed).

A Mann-Whitney $U$ test showed that pupil size did not differ significantly between the 3 groups either in the rest condition or in the stress condition. It is interesting, however, that the trend was for the 'emotional' group to have the smallest pupil size and to show the smallest change under stress, while the control group had the largest 
TABLE III

Mean Pupil Size and Range (mm) for Three Groups under Conditions of Rest and of Stress and Difference Between Conditions

\begin{tabular}{|c|c|c|c|c|c|c|c|c|c|c|}
\hline & \multirow{2}{*}{\multicolumn{2}{|c|}{ Group }} & & & \multicolumn{2}{|c|}{ Rest Condition } & \multicolumn{2}{|c|}{ Stress Condition } & \multirow{2}{*}{ Difference } & \multirow{2}{*}{$\underset{\text { Level }}{\text { Significance }}$} \\
\hline & & & & & Mean Value & Range & Mean Value & Range & & \\
\hline
\end{tabular}

pupil size and showed the largest change under stress. These results support those of Rubin et al. (1967), in that the trend was for their experimental group to have a smaller pupil size and to show smaller changes under stress than their control group.

In order to assess if there were significant differences between the groups with regard to the ratio of dilatation to constriction, calculations were based on the ratio of the following measurements. (i) After 1 minute dark (rest condition) and after 10 seconds light; (ii) after 5 minutes dark and after 10 seconds light (second application); (iii) after 1 minute stress and after 10 seconds light (second application).

Mean values for these calculations for the three groups can be seen in Table IV, and Table V shows the significance levels when comparisons are made between the groups. A Mann-Whitney U test was used for this analysis.

As can be seen in Table IV, the 'emotional'

TABLE IV

Mean Values for Calculations (i), (ii), and (iii) (see text) for 3 Groups

\begin{tabular}{l|c|c|c}
\hline \multicolumn{1}{c|}{ Group } & (i) & (ii) & (iii) \\
\hline RAP & $1 \cdot 83$ & $2 \cdot 16$ & $2 \cdot 22$ \\
Emotional & $1 \cdot 83$ & $2 \cdot 12$ & $2 \cdot 14$ \\
Control & $1 \cdot 94$ & $2 \cdot 35$ & $2 \cdot 38$ \\
& & & \\
\hline
\end{tabular}

\section{TABLE V}

Significance Levels when Comparisons are Made Between Experimental Groups and Control Group for Calculations (i), (ii), and (iii) (see text)

\begin{tabular}{c|l|l}
\hline \multicolumn{1}{c|}{ Calculation } & \multicolumn{1}{|c|}{ Group } & Significance Level \\
\hline (i) & Control v RAP & NS \\
(ii) & Control v Emotional & NS \\
& Control v RAP & NS \\
(iii) & Control v Emotional & $\mathbf{P}<0.05$ \\
& Control v RAP & NS \\
& Control v Emotional & $\mathrm{P}<0.02$ \\
\hline
\end{tabular}

group again showed the smallest change (except for calculation (i) when it was identical with the recurrent abdominal pain group) and the control group showed the largest change. When the experimental groups were compared with the control group a significant difference was found between the 'emotional' group and control group in respect of (ii) and (iii). The recurrent abdominal pain (RAP) group was not significantly different from the control group in any calculation.

Recovery, or constriction, after stress. Table VI shows the mean pupil size for the 3 groups at the end of the 10-minute recovery period. It shows also the mean values for the 1minute rest condition, so that the 2 sets of figures can be easily compared. A Wilcoxon matchedpairs signed-ranks test showed that the 'emotional'

TABLE VI

Mean Pupil Size (mm) for 3 Groups at End of 1-minute Rest Condition and 10-minute Recovery Period and Difference between Conditions

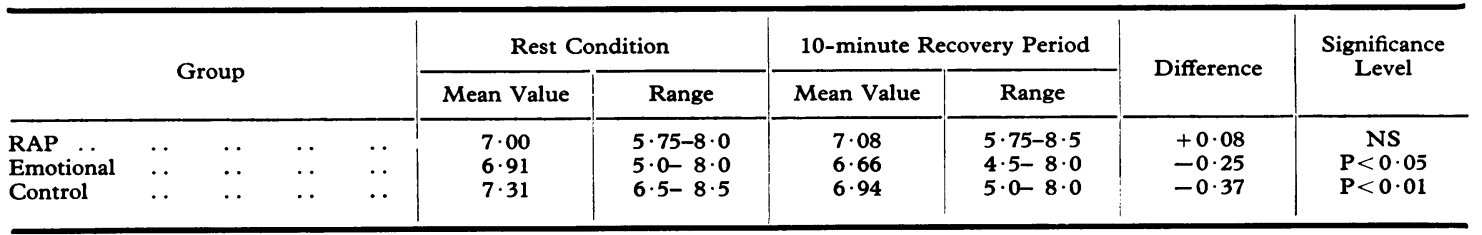


group and control group recovered to a level of constriction significantly below that of the 1minute rest condition $(P<0.05$ and $P<0.01$ respectively, two-tailed). The RAP group, however, did not return to their resting level, though the difference of $0.08 \mathrm{~mm}$ is not statistically significant.

The pupillary responses after stress were classified into 3 main groups: (a) constriction in approximately 1 to 2 minutes; (b) constriction in approximately 5 to 6 minutes or longer; and (c) constriction followed by dilatation, or dilatation followed by constriction. These were called 'rapid recovery', 'delayed recovery', and 'unstable recovery' respectively, and children were classified according to their mode of response.

Table VII shows the number of children in each

\section{TABLE VII}

Number of Children in Each Group whose Recovery From Stress was Rapid, Delayed, or Unstable

\begin{tabular}{l|c|c|c|c}
\hline Group & $\begin{array}{c}\text { Rapid } \\
\text { Recovery }\end{array}$ & $\begin{array}{c}\text { Delayed } \\
\text { Recovery }\end{array}$ & $\begin{array}{c}\text { Unstable } \\
\text { Recovery }\end{array}$ & Total \\
\cline { 2 - 5 } RAP & 0 & 6 & 10 & 16 \\
Emotional & 1 & 4 & 9 & 14 \\
Control & 10 & 6 & 4 & 20 \\
\hline
\end{tabular}

diagnostic group falling in each of the 3 categories. When a $\chi^{2}$ test was applied to the RAP group and the control group $\chi^{2}=12 \cdot 20 \quad(P<0 \cdot 005)$, and when applied to the 'emotional' group and control group $\chi^{2}=8.71(P<0.025)$. When the 2 experimental groups were combined to form 1 group $\chi^{2}=16 \cdot 87(\mathrm{P}<0 \cdot 001)$.

\section{Conclusions}

The investigation confirms that normal pupillary reactions are modified in children with psychosomatic disorder (recurrent abdominal pain with no physical cause) or with emotional disorder.

A change in autonomic activity is shown by the occurrence of unstable pupil recovery after stress in patients with these conditions, as compared with controls. Moreover, the control group was the only one which showed a significant difference in pupil size between rest and stress.

A difference in autonomic function as between psychosomatic and emotional disorders is indicated by the observation that after stress in the RAP group pupil size did not return to the resting level during the period of the investigations, while pupil size in the other groups did so and indeed became smaller. There was also a trend throughout for pupil size to be smallest in the emotional group.

Disturbances of autonomic function merit further investigation in psychosomatic and emotional disorders, both as a method of diagnosis and to help elucidate the underlying mechanisms whereby symptoms may be produced.

Our thanks are due to Messrs. Kodak Ltd., who assisted one of us (C.G.T.) with a grant towards the expenses of the project.

\section{REFERENCES}

Apley, J. (1964). The Child with Abdominal Pains. Blackwell, Oxford.

Rubin, L. S., Barbero, G. J., and Sibinga, M. S. (1967). Pupillary reactivity in children with recurrent abdominal pain. Psychosomatic Medicine, 29, 111.

Correspondence to Dr. John Apley, Bristol Royal Hospital for Sick Children, St. Michael's Hill, Bristol 2. 